\title{
Entanglement of Remote Atomic Qubits
}

\author{
D. N. Matsukevich, T. Chanelière, S. D. Jenkins, S.-Y. Lan, T. A. B. Kennedy, and A. Kuzmich \\ School of Physics, Georgia Institute of Technology, Atlanta, Georgia 30332-0430, USA
}

(Received 2 November 2005; published 25 January 2006)

\begin{abstract}
We report observations of entanglement of two remote atomic qubits, achieved by generating an entangled state of an atomic qubit and a single photon at site $A$, transmitting the photon to site $B$ in an adjacent laboratory through an optical fiber, and converting the photon into an atomic qubit. Entanglement of the two remote atomic qubits is inferred by performing, locally, quantum state transfer of each of the atomic qubits onto a photonic qubit and subsequent measurement of polarization correlations in violation of the Bell inequality $|S| \leq 2$. We experimentally determine $S_{\exp }=2.16 \pm 0.03$. Entanglement of two remote atomic qubits, each qubit consisting of two independent spin wave excitations, and reversible, coherent transfer of entanglement between matter and light represent important advances in quantum information science.
\end{abstract}

DOI: 10.1103/PhysRevLett.96.030405

Realization of massive qubits, and their entanglement, is central to practical quantum information systems [1-3]. Remote entanglement of photons can now be achieved in a robust manner using the well-developed technology of spontaneous parametric down-conversion [4], with propagation to remote locations by means of optical fibers. Photons, however, are difficult to store for any appreciable period of time, whereas qubits based on ground-state atoms have long lifetimes. Local entanglement of massive qubits has been observed between adjacent trapped ions [5] and between pairs of Rydberg atoms in a collimated beam [6]. In order to entangle qubits at remote locations, the use of photons as an intermediary seems essential [7-10]. Photons also offer some flexibility as information carriers as they can propagate in optical fiber with low losses. The creation, transport, storage, and retrieval of single photons between remote atomic ensembles located in two different laboratories were recently observed [11] [see also a related work on electromagnetically induced transparency (EIT) with single photon pulses [12] ]. The first step in creating remote entanglement between massive qubits is to entangle one such qubit with the mediating light field, which is then directed towards the second qubit via an optical fiber. There have recently been important advances towards this goal by demonstrating entanglement of a photon with a trapped ion [13], with a collective atomic qubit $[14,15]$, and with a single trapped atom [16].

A promising route towards the creation and application of long-lived qubit entanglement in scalable quantum networks was proposed by Duan, Lukin, Cirac, and Zoller $[2,10]$. These atomic qubits rely on collective atomic states containing exactly one spin excitation. In two recent experiments, collective atomic qubits were generated using cold atomic ensembles [14,15]. In Ref. [14] the logical states were single spin wave excitations, one in each of two distinct atomic ensembles inside a high vacuum chamber [17]. In Ref. [15], two orthogonal spin waves in a single cold ensemble represented the logical qubit states. The experiments $[14,15]$ realized a single atomic qubit system,
PACS numbers: 03.65.Ud, 03.67.Mn, 42.50.Dv

but did not address the issue of entanglement of atomic qubits.

While remote entanglement of atomic qubits has not been previously demonstrated, Refs. $[14,15]$ realized two basic primitives of a quantum network: (a) entanglement of photonic and atomic qubits, and (b) quantum state transfer from an atomic to a photonic qubit. The crucial additional ingredient is the reverse operation, the conversion of a photonic qubit into an atomic qubit. This enables the transfer of atom-photon entanglement into remote atomic qubit entanglement.

Here we report remote atomic qubit entanglement using cold atomic clouds of ${ }^{85} \mathrm{Rb}$ confined at sites $A$ and $B$, as shown in Fig. 1. These sites are situated in separate laboratories and linked by an optical fiber. A notable distinction between the two nodes is that the qubit generated at site $A$ is written on an unpolarized atomic ensemble, as in Ref. [15], whereas at site $B$ the atomic ensemble is prepared, ideally, in the $(m=0)$ Zeeman state of the $F=2$ ground level by optical pumping. All the light fields responsible for trapping and cooling of the atoms, as well as the quadrupole magnetic fields at both sites, are shut off during the period of the protocol. The ambient magnetic field at each site is compensated by three pairs of Helmholtz coils, and a bias field of $0.2 \mathrm{G}$ is added at site $B$ for the purpose of optical pumping.

Our protocol starts with the generation of an entangled state of a signal photon and a collective atomic qubit at site $A$, achieved through Raman scattering of a classical laser write pulse. The state can be represented schematically as

$$
|\Psi\rangle=|0\rangle_{a}|0\rangle_{f}+\chi\left(\cos \eta|+\rangle_{a}|+\rangle_{f}+\sin \eta|-\rangle_{a}|-\rangle_{f}\right),
$$

where $|+\rangle_{f} \equiv \hat{a}_{+}^{\dagger}|0\rangle_{f}$ and $|-\rangle_{f} \equiv \hat{a}_{-}^{\dagger}|0\rangle_{f}$ are the normalized states of positive and negative helicity of the signal photon, $|0\rangle_{f}$ is the field vacuum state, $| \pm\rangle_{a} \equiv \hat{s}_{ \pm}^{\dagger}|0\rangle_{a}$ describes the two logical qubit states, corresponding to non- 


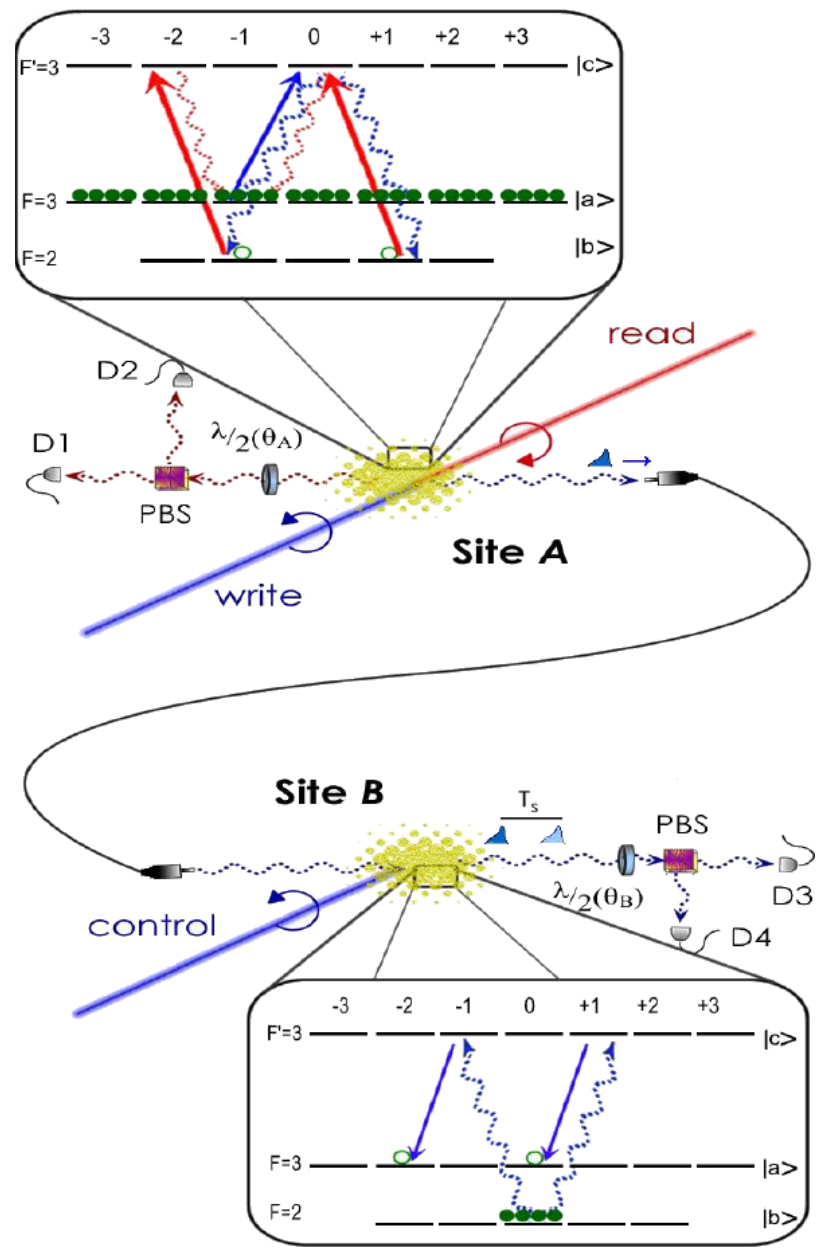

FIG. 1 (color online). A schematic diagram of our experimental setup. Two cold atomic ensembles of ${ }^{85} \mathrm{Rb}$, an unpolarized sample at site $A$, and a spin-polarized sample at site $B$, separated by $5.5 \mathrm{~m}$, are connected by a single-mode fiber. The insets show the structure and the initial populations of the atomic levels for the two ensembles; for simplicity only couplings to the $m=-1$ state of level $|a\rangle$ are shown at site $A$. An entangled state of a collective atomic qubit and a signal field (wavy blue line) is generated at site $A$ by Raman scattering of the write laser field (solid blue line). The orthogonal helicity states of the generated signal field are transmitted via optical fiber from site $A$ to site $B$, where they are converted to orthogonal collective atomic excitations, stored for a duration $T_{s}$, and subsequently converted into idler field $B$ (wavy blue line) by adiabatic variation of the control field amplitude. The atomic qubit at site $A$ is similarly converted into idler $A$ (wavy red line) by a read laser pulse, counterpropagating with respect to the write pulse. For polarization analysis, each idler field propagates through a quarterwave plate (not shown), a half-wave plate $(\lambda / 2)$, and a polarizing beam splitter (PBS). Polarization correlations of the idler fields are recorded by photoelectric detection using the single photon detectors $D 1-D 4$.

symmetric collective atomic modes [18], $|0\rangle_{a}$ represents the collective atomic ground state, and $\chi \ll 1$. The asymmetry angle $\eta=0.81 \pi / 4$ [15]. Equation (1) represents probabilistic entanglement generation, where ideally for each signal photon emission event, an entangled atomic qubit is created in the atomic ensemble [2,13]. Since we deal with an unpolarized atomic ensemble, the state of the system is more rigorously described by a density operator as discussed in Ref. [15].

The orthogonal polarization modes of the signal field produced at site $A$ are directed along the optical fiber to site $B$ [19]. The signal field propagation in the atomic medium at site $B$ is controlled by an additional laser field (control) through the process of EIT [20-25]. We implement the storage phase at site $B$, by adiabatically reducing the control field amplitude to zero, while the signal pulse lies within the cloud. The orthogonal atomic spin wave excitations thereby created in the spin-polarized gas constitute the logical states of the atomic qubit. In order to convert the signal field qubit into a collective atomic qubit, it is necessary that the optically thick atomic sample supports EIT for both field helicities [23]. To this end, we optically pump the atomic cloud at Site $B$ using a linearly polarized field resonant to the $F=2 \leftrightarrow F^{\prime}=2$ transition of the $D_{1}$ line, and an additional repumping field resonant to the $F=3 \leftrightarrow F^{\prime}=3$ transition of the $D_{2}$ line. We measured the optical thickness $d \simeq 8$ for both circular components of the signal field.

By switching off the control field over a period of about $20 \mathrm{~ns}$, the photonic qubit is converted into an atomic qubit. At this stage remote atomic qubits should have been created at sites $A$ and $B$. Atoms at site $B$ should, ideally, be prepared in a single Zeeman $m=0$ state of the $F=2$ hyperfine ground level (lower inset of Fig. 1). In practice the pumping is not perfect, possibly due to radiation trapping. We measure lower storage and retrieval efficiency for the negative helicity signal component compared with that of the positive helicity component ( $3 \%$ vs $8 \%$ ). Numerical simulations indicate that the discrepancy between the efficiencies is consistent with a residual population in the $|F=2, m=-2\rangle$ atomic state at the $10 \%$ level [26]. This results in undesirable absorption of the signal field with negative helicity.

The signal photon of helicity $\alpha= \pm 1$ is stored in the ensemble at site $B$ with efficiency $\epsilon_{\alpha}$. After a storage time $T_{s}$, the nonvacuum component of the state of the two ensembles is given by the following density operator: $\hat{\rho}=$ $(1-\epsilon) \hat{\rho}_{A}+\epsilon \hat{\rho}_{A B}$, where the component $\hat{\rho}_{A}$ describes the state of single excitation at site $A$, and is expressed by

$$
\begin{aligned}
\hat{\rho}_{A}= & \frac{1-\epsilon}{1-\epsilon} \cos ^{2} \eta \hat{s}_{A+}^{\dagger} \hat{\rho}_{\mathrm{vac}} \hat{s}_{A+} \\
& +\frac{1-\epsilon_{+}}{1-\epsilon} \sin ^{2} \eta \hat{s}_{A-}^{\dagger} \hat{\rho}_{\mathrm{vac}} \hat{s}_{A-},
\end{aligned}
$$

where $\hat{\rho}_{\text {vac }}$ is the product of the ground-state atomic density operators for the ensembles at sites $A$ and $B$. The density operator $\hat{\rho}_{A B}=\hat{\Psi}_{A B}^{\dagger}\left(T_{s}\right) \hat{\rho}_{\text {vac }} \hat{\Psi}_{A B}\left(T_{s}\right)$ in the twoqubit subspace represents an entangled atomic state where

$$
\hat{\Psi}_{A B}^{\dagger}\left(T_{s}\right)=e^{i \phi\left(T_{s}\right)} \cos \eta^{\prime} \hat{s}_{A+}^{\dagger} \hat{s}_{B-}^{\dagger}-\sin \eta^{\prime} \hat{s}_{A-}^{\dagger} \hat{s}_{B+}^{\dagger}
$$


with $\cos \eta^{\prime}=\sqrt{\epsilon_{-} / \epsilon} \cos \eta$, and $\epsilon=\epsilon_{-} \cos ^{2} \eta+\epsilon_{+} \sin ^{2} \eta$ is the average efficiency of photon storage at site $B$. The phase $\phi(t)=-2\left(g \mu_{B} / \hbar\right) B_{0} t$ is induced by the applied magnetic field $B_{0}=0.2 \mathrm{G}$ oriented along the propagation axis at site $B$, where $g$ is the Landé $g$ factor for hyperfine level with $F=3$.

Ideally, entanglement should have been created between the collective atomic qubits at sites $A$ and $B$. After a storage time $T_{s}$, the remote collective atomic excitations are converted by quantum state transfer into idler fields emanating from sites $A$ and $B$, using a read laser pulse at site $A$ and by reactivating the control field at site $B[14,15]$. We note that when we produce the signal and idler fields under conditions of phase-matching collective enhancement of the atom-field interaction causes the atom to return to its initial state at the end of the write-read cycle. We emphasize that this is true even for a statistical mixture of Zeeman states, as at site $A$. The resulting idler-idler photoelectric correlations may be calculated using the effective two-photon state

$$
\left|\Psi_{2}\right\rangle=\cos \eta_{f}|H V\rangle+e^{i \phi_{f}} \sin \eta_{f}|V H\rangle,
$$

where $|H V\rangle=\hat{a}_{A, H}^{\dagger} \hat{a}_{B, V}^{\dagger}|0\rangle_{f}$ and $|V H\rangle=\hat{a}_{A, V}^{\dagger} \hat{a}_{B, H}^{\dagger}|0\rangle_{f}$, and the subscripts $A$ and $B$ indicate the idler mode at the respective site. We omit higher-order terms in photon number [11].

The phase $\phi_{f}$, which includes the contributions due to the Larmor precession $\phi\left(T_{s}\right)$, the light phase shifts in the atomic media, and various optical elements, is introduced as an adjustable parameter. The mixing angle $\eta_{f}$ is determined by the relative efficiencies with which the two-qubit states are transferred from the atomic ensembles to the idler fields. If we assume equal transfer efficiencies at site $A$, we find $\cos \eta_{f}=\sqrt{\epsilon_{B-} / \epsilon_{B}} \cos \eta$, where $\epsilon_{B}=$ $\epsilon_{B-} \cos ^{2} \eta+\epsilon_{B+} \sin ^{2} \eta$ and $\epsilon_{B \pm}$ is the combined storage and retrieval efficiency for a photon of helicity \pm at site $B$. Measurements of these efficiencies give $\epsilon_{B+}=0.08$ and $\epsilon_{B-}=0.03$. With $\eta=0.81 \pi / 4$ fixed by the atom-photon entanglement process at site $A$ [15] we get $\eta_{f}=1.12 \pi / 4$. Our experimental data, including those displayed in Fig. 3 below, are consistent with this value of $\eta_{f}$ and $\phi_{f} \ll 1$.

The above arguments are clearly conditional on the generation of the signal qubit. According to Eq. (1), the corresponding probability scales as $\chi^{2}$, and this determines the efficiency of the probabilistic entanglement generation. However, as Duan et al. point out [2], quantum network protocols eliminate the vacuum component of Eq. (1) and only the entanglement characteristics of the nonvacuum component of $|\Psi\rangle$ are relevant [27]. In our experiment, atomic qubits were stored for a time $500 \mathrm{~ns}$ at site $A$ and $200 \mathrm{~ns}$ at site $B$. It should be possible to extend the qubit storage times to longer than $10 \mu \mathrm{s}$, as the single-quanta storage results suggest [11].

The measurement of the atomic qubits is performed by quantum state transfer onto the idler fields at both sites, using the read laser pulse at site $A$ and the control laser pulse at site $B$. The polarization state of either idler field is measured using a polarizing beam splitter and two single photon detectors, $D 1, D 2$ for site $A$ and $D 3, D 4$ for site $B$ (additional technical details are given in Refs. $[11,14,15]$ ). Polarization correlations between the idler fields produced at the remote sites are recorded and analyzed for the presence of entanglement. The contributions of the vacuum and single photon idler excitations are excluded in the observed photoelectric coincidences between the remote sites [27]. Since quantum state transfer is a local process, it cannot generate entanglement. Hence, observation of idler field entanglement confirms probabilistic entanglement of the two remote atomic qubits. We denote the number of such coincidences between detector $D n, n=1,2$ at site $A$ and detector $D m, m=3,4$ at site $B$ by $C_{n m}\left(\theta_{A}, \theta_{B}\right)$. Here $\theta_{A}$ and $\theta_{B}$ are the angles by which polarization is rotated by the half-wave plates at these sites.

The two-particle interference produces a high-visibility sinusoidal fringe pattern for the coincidence rates $C_{n m}\left(\theta_{A}, \theta_{B}\right)$, which is characteristic of entangled particles. Figure 2 shows measured coincidence fringes for some representative angles. We calculate the coincidence rates $C_{n m}\left(\theta_{A}, \theta_{B}\right)$ to be

$$
\begin{aligned}
C_{13}\left(\theta_{A}, \theta_{B}\right) \propto & \xi_{1} \xi_{3} \mid\left(\cos \eta_{f}+e^{i \phi_{f}} \sin \eta_{f}\right) \sin \left(\theta_{B}+\theta_{A}\right) \\
& +\left.\left(\cos \eta_{f}-e^{i \phi_{f}} \sin \eta_{f}\right) \sin \left(\theta_{B}-\theta_{A}\right)\right|^{2},
\end{aligned}
$$

where $\xi_{m}$ is the overall efficiency (including propagation losses) for detector $D_{m}$, and similar expressions for the other three rates [26].

In order to confirm two-particle entanglement, we check Bell inequality violation. This involves measurement of discrete values of $C_{n m}\left(\theta_{A}, \theta_{B}\right)$ on the slopes of the fringe pattern. Explicitly, following Ref. [28], we calculate $E\left(\theta_{A}, \theta_{B}\right)$, given by

$$
\frac{C_{13}\left(\theta_{A}, \theta_{B}\right)+C_{24}\left(\theta_{A}, \theta_{B}\right)-C_{14}\left(\theta_{A}, \theta_{B}\right)-C_{23}\left(\theta_{A}, \theta_{B}\right)}{C_{13}\left(\theta_{A}, \theta_{B}\right)+C_{24}\left(\theta_{A}, \theta_{B}\right)+C_{14}\left(\theta_{A}, \theta_{B}\right)+C_{23}\left(\theta_{A}, \theta_{B}\right)} .
$$

In Fig. 3 we display $E\left(\theta_{A}, \theta_{B}\right)$ as a function of $\theta_{A}$, for four values of $\theta_{B}$. By fitting the correlation functions in

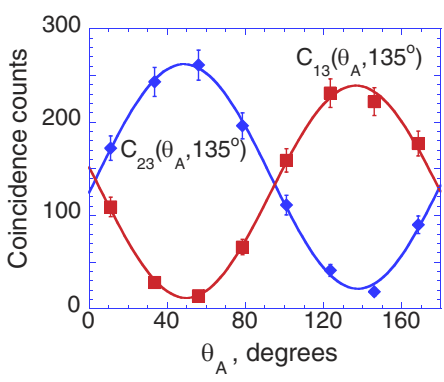

FIG. 2 (color online). Measured coincidence fringes $C_{n 3}\left(\theta_{A}, \theta_{B}\right)$ as a function of $\theta_{A}$, for $\theta_{B}=135^{\circ} ; n=1$, diamonds; $n=2$, squares. The curves are sinusoidal fits to the data. Each point is acquired for $15 \mathrm{~min}$. The effective repetition rate is $108 \mathrm{kHz}$, and each trial takes $1.1 \mu \mathrm{s}$. 
(a)

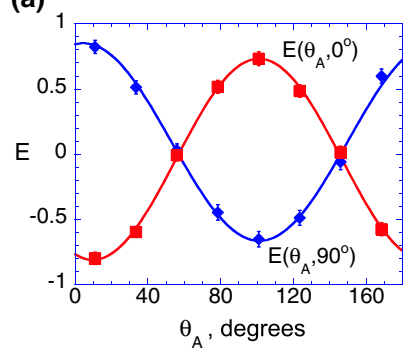

(b)

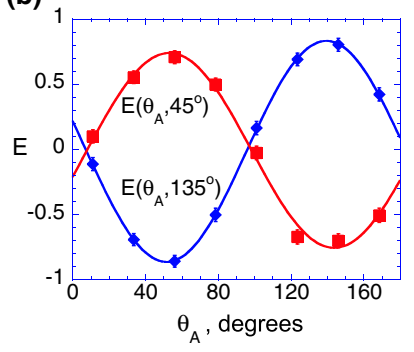

FIG. 3 (color online). Measured correlation function $E\left(\theta_{A}, \theta_{B}\right)$ as a function of $\theta_{A}$. (a) $\theta_{B}=0^{\circ}$, squares; and $90^{\circ}$, diamonds. (b) $\theta_{B}=45^{\circ}$, squares,; and $135^{\circ}$, diamonds. The curves are sinusoidal fits to the data.

Fig. 3 with sinusoids, we determine a set of four pairs of angles $\theta_{A}=78.5^{\circ}, \theta_{B}=45^{\circ}, \theta_{A}^{\prime}=33.5^{\circ}$, and $\theta_{B}^{\prime}=0^{\circ}$ that should maximize the Bell inequality violation. We acquire data for two hours at each of these four points (Table I). In order to account for unequal efficiencies of the detectors $D 1, D 2$ and $D 3, D 4$, each correlation measurement consisted of four runs, flipping polarization of either one of the idler fields by $90^{\circ}$ between the runs. As a result, the products $\xi_{m} \xi_{n}$ are effectively replaced by the symmetric factor $\frac{1}{4}\left(\xi_{1}+\xi_{2}\right)\left(\xi_{3}+\xi_{4}\right)$ in Eq. (5). In this case the correlation function $E\left(\theta_{A}, \theta_{B}\right)$ becomes independent of these efficiencies:

$$
\begin{aligned}
E\left(\theta_{A}, \theta_{B}\right)= & -\frac{1}{2}\left\{\cos \left[2\left(\theta_{A}-\theta_{B}\right)\right]\left(1-\cos \phi_{f} \sin 2 \eta_{f}\right)\right. \\
& \left.+\cos \left[2\left(\theta_{A}+\theta_{B}\right)\right]\left(1+\cos \phi_{f} \sin 2 \eta_{f}\right)\right\} .
\end{aligned}
$$

The Bell inequality [28] is then $|S| \leq 2$, where $S=$ $E\left(\theta_{A}, \theta_{B}\right)+E\left(\theta_{A}^{\prime}, \theta_{B}\right)+E\left(\theta_{A}, \theta_{B}^{\prime}\right)-E\left(\theta_{A}^{\prime}, \theta_{B}^{\prime}\right)$. We find $S=2.16 \pm 0.03 \not \leq 2$, in clear violation of the Bell inequality. No corrections for background or dark counts were made to any of the experimental counting rates, and these are chiefly responsible for the reduction in the observed value of $S$ from the ideal value of 2.60 predicted by our theoretical model [26]. In conclusion, we have demonstrated entanglement of two remote atomic qubits, based on collective atomic states. By photoelectric detection of polarization correlations of the idler fields we have also confirmed the mapping of atomic qubit entanglement onto

TABLE I. Measured values of the correlation function $E\left(\theta_{A}, \theta_{B}\right)$ at particular polarization settings and the Bell parameter $S$.

\begin{tabular}{ccr}
\hline \hline$\theta_{A}\left({ }^{\circ}\right)$ & $\theta_{B}\left({ }^{\circ}\right)$ & $E\left(\theta_{A}, \theta_{B}\right)$ \\
\hline 78.5 & 45 & $0.447 \pm 0.017$ \\
33.5 & 45 & $0.640 \pm 0.014$ \\
78.5 & 0 & $0.572 \pm 0.015$ \\
33.5 & 0 & $-0.504 \pm 0.016$ \\
& & $S=2.16 \pm 0.03$ \\
\hline
\end{tabular}

photonic qubits. Long-lived entanglement of remote massive qubits and entanglement transfer between matter and light are important prerequisites for realization of a scalable quantum information network.

This work was supported by Office of Naval Research, NASA, National Science Foundation, Research Corporation, Alfred P. Sloan Foundation, and Cullen-Peck Foundation. We thank M.S. Chapman for illuminating discussions and E.T. Neumann for experimental assistance.

[1] H.-J. Briegel et al., Phys. Rev. Lett. 81, 5932 (1998).

[2] L.-M. Duan et al., Nature (London) 414, 413 (2001).

[3] A. K. Ekert, Phys. Rev. Lett. 67, 661 (1991).

[4] D. Bouwmeester et al., Nature (London) 390, 575 (1997); D. Boschi et al., Phys. Rev. Lett. 80, 1121 (1998); M. Aspelmeyer et al., Science 301, 621 (2003).

[5] C. A. Sackett et al., Nature (London) 404, 256 (2000).

[6] E. Hagley et al., Phys. Rev. Lett. 79, 1 (1997).

[7] C. Cabrillo et al., Phys. Rev. A 59, 1025 (1999).

[8] S. Bose et al., Phys. Rev. Lett. 83, 5158 (1999).

[9] A. S. Sørensen and K. Mølmer, Phys. Rev. Lett. 90, 127903 (2003).

[10] L.-M. Duan, Phys. Rev. Lett. 88, 170402 (2002).

[11] T. Chanelière et al., Nature (London) 438, 833 (2005).

[12] M. Eisaman et al., Nature (London) 438, 837 (2005).

[13] B. B. Blinov et al., Nature (London) 428, 153 (2004).

[14] D. N. Matsukevich and A. Kuzmich, Science 306, 663 (2004).

[15] D. N. Matsukevich et al., Phys. Rev. Lett. 95, 040405 (2005).

[16] M. Weber, Ph.D. thesis, University of Munich, 2005; J. Volz et al., quant-ph/0511183.

[17] A similar experiment to Ref. [14] reported the use of ensembles in two different vacuum chambers within the same room: C. W. Chou et al., Nature (London) 438, 828 (2005).

[18] A. Kuzmich and T. A. B. Kennedy, Phys. Rev. Lett. 92, 030407 (2004).

[19] The fiber channel was polarization stable over periods of days - much longer than the data acquisition times. As the signal field propagates from site $A$ to site $B$, it passes through two quarter-wave plates, causing the transformation of the signal field operators $\hat{a}_{ \pm} \rightarrow \pm \hat{a}_{\mp}$.

[20] M. O. Scully and M.S. Zubairy, Quantum Optics (Cambridge University Press, Cambridge, England, 1997).

[21] S. E. Harris, Phys. Today 50, No. 7, 36 (1997).

[22] L. V. Hau et al., Nature (London) 397, 594 (1999).

[23] M. Fleischhauer and M. D. Lukin, Phys. Rev. Lett. 84, 5094 (2000).

[24] D. F. Phillips et al., Phys. Rev. Lett. 86, 783 (2001).

[25] C. Liu et al., Nature (London) 409, 490 (2001).

[26] S. D. Jenkins et al. (to be published).

[27] S. J. van Enk and H.J. Kimble, Science 309, 1187b (2005); D. N. Matsukevich and A. Kuzmich, ibid. 309, $1187 \mathrm{c}$ (2005).

[28] J. F. Clauser et al., Phys. Rev. Lett. 23, 880 (1969). 